COMMENT. Seizures in Dravet syndrome (DS) begin in the first year, usually at or around 6 months of age. Early seizures are typically prolonged and associated with fever or infection. Known seizure triggers include, in addition to fever and infection, hot water bath, and photic or pattern stimulation. Mutations in the SCN1A gene are found in $67 \%$ to $86 \%$ of DS cases. (Dravet C et al, 1982, 2005; Millichap JJ, Koh S, Laux LC, Nordli DR Jr. Dravet syndrome. When to suspect the diagnosis. Neurology 2009;73:1-1).

Wiznitzer M, in an editorial, (Lancet Neurol 2010;9:559-561) comments that the study by McIntosh and colleagues has limitations but it attempts to introduce science over speculation and bias in the question and controversy of pertussis vaccination and encephalopathy. Although 12 individuals with DS and a first seizure within 2 days of whole cell or acellular DTP had earlier onset than the 28 with a first seizure before or more distant from vaccination, their clinical outcome, including cognitive disability, was not different, and no worsening occurred in individuals who received further vaccinations. Both authors conclude that outcome is determined by the underlying disorder and not by proximity to vaccine administration.

\title{
KETOGENIC DIET FOR EPILEPSY WITH TYPE 1 DIABETES
}

Researchers at Medical University Vienna, Austria, report the efficacy and safety of the ketogenic diet (KD) in treatment of epilepsy in a 3-year 6 month-old girl with diabetes type 1 followed for 15 months. Right-sided spastic hemiparesis with widespread left hemisphere malformation were diagnosed at 9 months and were associated with focal tonic seizures during sleep. EEG-video monitoring showed interictal spikes in left central-parietal, and frontal and occipital electrodes. Seizure frequency (1-2 per week) increased at 30 months of age and because of side effects, the parents stopped antiepileptic drug treatment but agreed to a trial of the KD. John Hopkins' KD protocol was followed with a 2.5:1 ratio, and insulin requirement was reduced. Since the KD, no clinical seizures were reported but after 10 months, the EEG showed persistent epileptiform activity over the left hemisphere and 4 subclinical seizures, especially during sleep. Gains in language and motor skills were observed. No severe hypoglycemia or ketoacidosis occurred and metabolic control was maintained. The child refused ketogenic meals and the parents discontinued the diet after 15 months, and no clinically overt seizures were reported. (Dressler A, Reichofer E, Trimmel-Schwahofer P et al. Type 1 diabetes and epilepsy: efficacy and safety of the ketogenic diet. Epilepsia June 2010;51:1086-1089). (Respond: E-mail: anastasia.dressler@meduniwien.ac.at).

COMMENT. The authors conclude that diabetes does not preclude the use of the ketogenic diet in treatment of childhood epilepsy. Glycemic control is maintained without severe side effects, and development may be improved. 